\title{
Mystery of bilateral breast masses
}

\section{Nausheen Khan, FC Rad \\ Zaeem I Ebrahim, FC Rad Irma van de Werke, FRCR}

Department of Radiology, Kalafong Hospital and University of Pretoria

Corresponding author: Z Ebrahim (zaeem@iafrica.com)

\section{Introduction}

Leiomyosarcoma (LMS) is an uncommon malignant tumour of smooth muscle origin. It arises in the gastro-intestinal tract, retroperitoneum, urinary bladder, uterus and soft tissue. Peritoneal leiomyosarcomatosis $(\mathrm{PL})$ is defined as a peritoneal dissemination of a primary sarcoma. We present a case of leiomyosarcomatosis with widespread dissemination including involvement of both breasts.

\section{Case report}

A 38-year-old woman, retrovirus negative, presented at the outpatient department of Kalafong Hospital, with a 3-month history of weight loss and enlarging bilateral breast masses. On examination there were numerous large, hard, nodular fixed masses in both breasts and axillae. The preliminary diagnosis at this stage was lymphoma, bilateral breast carcinoma, or unknown primary malignancy with metastasis to the axillae and breasts. On further examination, masses were felt in the anterior abdominal wall and in the back and thigh. Several soft tissue core biopsies were done of the axillary masses. A cervical smear showed no malignant cells.

Computed tomography (CT) of the chest and abdomen demonstrated a large complex mass arising from the uterus with both cystic and solid components (Fig. 1). Another mass lesion was noted in the right kidney with extension into the inferior vena cava with complete occlusion (Fig. 2). There were several soft-tissue enhancing masses in both breasts and axillae (Fig. 3); a mass in the anterior abdominal wall (Fig. 4); and a mass in the pericardium (Fig. 5) and the posterior compartment of the left thigh (Fig. 6). All the masses had similar soft-tissue features. The diagnosis on CT was disseminated leiomyosarcomatosis. Histological examination of the tissue submitted showed several cores of tumour comprising pleomorphic spindle-shaped cells. Tumour giant cells were also noted and several mitoses were present including atypical mitosis. There were areas of myxoid degeneration. Immunohistochemical staining of the tissue was diffusely and strongly positive for actin, negative for desmin, and negative for cluster of differentiation (CD) of 117. The histological and immunohistochemical features were most in keeping with the diagnosis of a leiomyosarcoma. Further evaluation of the uterine mass was recommended and discussed with the patient and her prognosis explained. She refused further intervention. She was sent home with the clinical diagnosis of disseminated leiomyosarcomatosis.

\section{Discussion}

Leiomyosarcoma is a relatively uncommon malignant mesenchymal tumour that exhibits smooth muscle differentiation. ${ }^{1,2}$ It is a tumour of adult life seen more commonly in women than men, and generally has a poor prognosis with a high percentage of recurrence. Leiomyosarcomatosis is defined as dissemination of leimyosarcoma

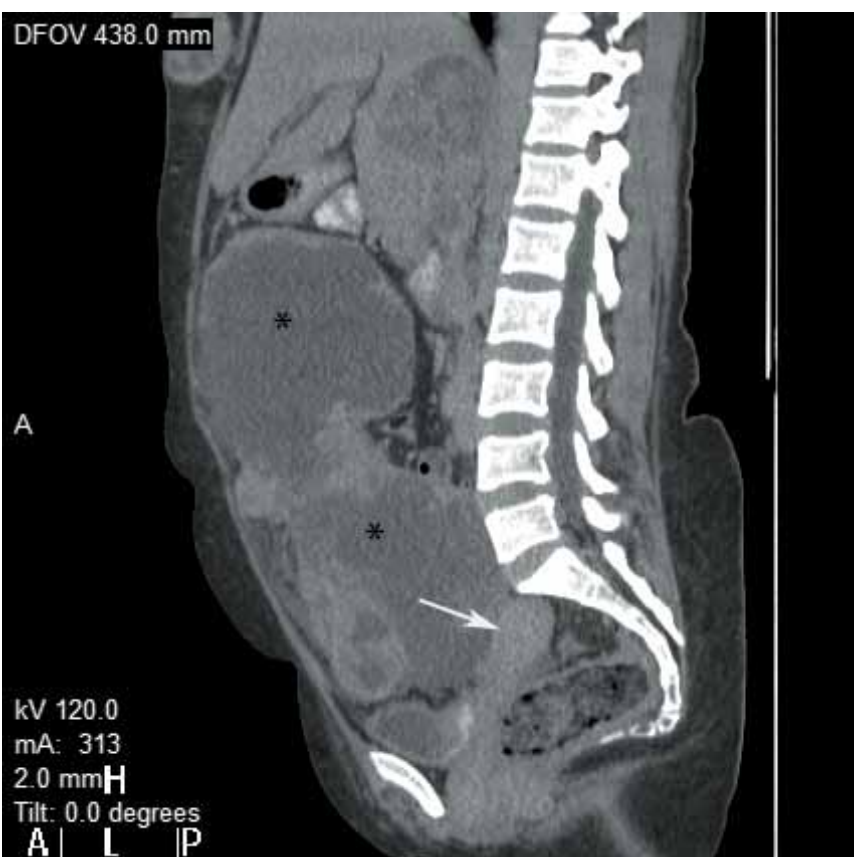

Fig. 1. Sagittal post-contrast CT through the pelvis demonstrates a large hetrogenous mass with areas of low densities representing necrosis (asterisks) arising from the anterior superior aspect of the uterus. Arrow points to the uterus.

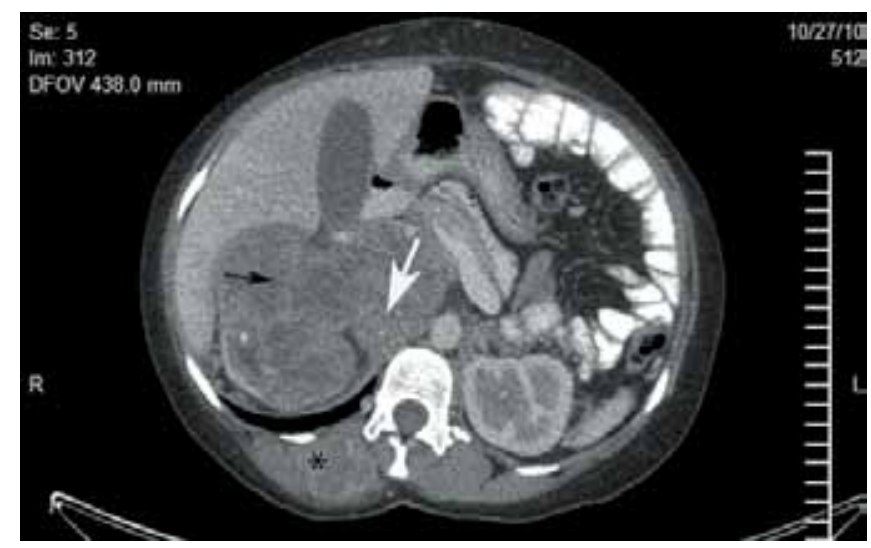

Fig. 2. Axial CT post-contrast through the upper abdomen. There is a large mass in the right kidney (open arrow) with complete occlusion of the IVC (black arrow). There is also a soft-tissue mass in the right paraspinal mass (asterisk).

from a primary in the gastro-intestinal tract to the peritoneum or omentum; or haematogenous spread to distant sites including the head and neck, skin and soft tissue. ${ }^{2}$ Diffuse peritoneal and omental seeding are well-known forms of dissemination of metastatic carcinoma either via direct invasion through the serosa, or haematogenous or embolic spread. ${ }^{2,3}$ Breast metastasis from extramammary malignancy is rare, constituting $2 \%$ of breast tumours. ${ }^{4}$ Conforming to Virchow's concept, the breast has resistance to metastasis from extra-mammary malignancy because the breast has large areas of fibrous tissue, with a relatively poor blood supply. ${ }^{4,5}$ Breast metastasis with multiple lesions from uterine leiomyosarcoma and especially with bilateral involvement as seen in our case, is less common, however. Primary leiomyosarcoma of the breast 


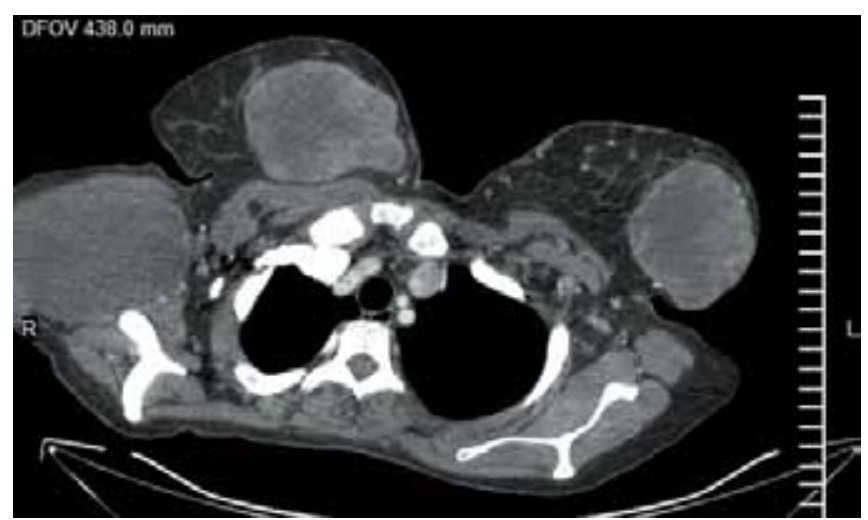

Fig. 3. Axial CT post-contrast through the chest at the level of the breasts. There are bilateral breast masses and a mass in the right axilla.

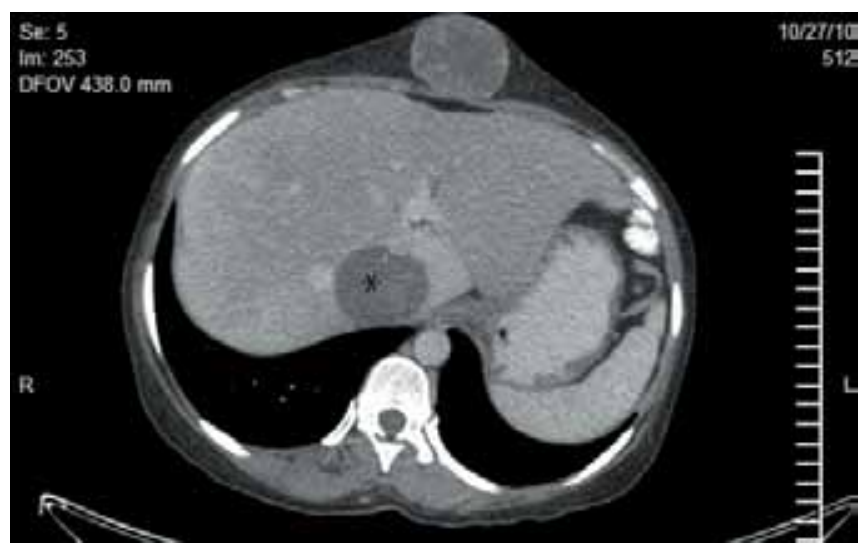

Fig. 4. Solitary mass in the anterior abdominal wall. Note a markedly dilated IVC (asterisk).

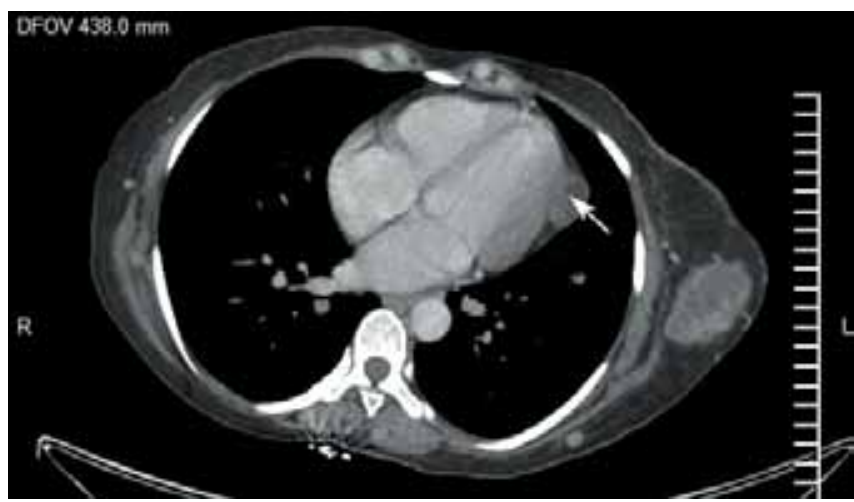

Fig. 5. Mass in the pericardium (arrow) and left axilla.

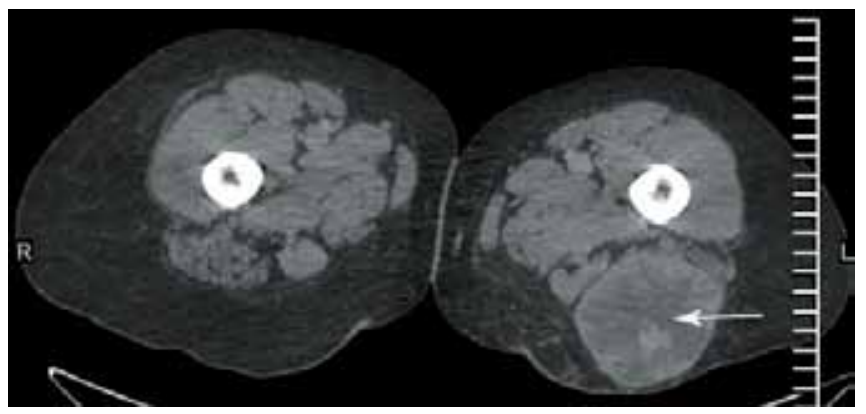

Fig. 6. Similar soft tissue mass in the posterior compartment of the left thigh. is rarer ${ }^{4,6}$ it is usually low-grade and should be distinguished from leiomyoma. ${ }^{6}$ The average interval for occurrence of non-breast tumour metastasis to the breast is approximately 2 years. ${ }^{5.6}$ Our patient presented primarily with metastatic breast masses rather than with a primary tumour in the uterus. In $85 \%$ of patients, metastatic leiomyosarcoma presents as a solitary breast lump and only $15 \%$ as bilateral or multiple lumps as in our case. Leiomyomas and leiomyosarcomas arise from the spindle cells of the muscular coat of the gastro-intestinal tract and less commonly from the muscularis mucosa. ${ }^{7}$ CT examination in leiomyosarcomatosis usually demonstrates multiple dumbbell or oval tumours of soft-tissue density that enhance homogenously on intravenous contrast administration. Tumours more than $5 \mathrm{~cm}$ may show central hypodensity owing to necrosis. Calcifications are uncommon.?

\section{Conclusion}

Metastases to the breasts from primary sites are unusual and bilateral involvement even rarer. So far there have only been a few cases reported of leiomyosarcoma to the breast. These metastases to the breast are seen on an average interval of approximately 2 years after the detection of the primary site. In our case, the patient presented primarily with metastasis. The diagnosis becomes a formidable issue when the breast metastasis represents the first event in the patient's work-up. The presence of metastatic tumour in the breast carries a poor prognosis, and radical procedures are unnecessary and can be avoided.

1. Akata D, Aralasmak A, Ozmen MN, et al. US and CT findings of multicentric leiomyosarcomatosis. Eur Radiol1999;9:711-714.

2. Choi BNI, Lee WJ, Chi JG, Han JK. CT manifestations of peritoneal leiomyosarcomatosis. AJR 1990;10(155): 799-801.

3. Rha SU, Ha HK, Kim AY, et al. Peritoneal leiomyosarcomatosis originating from gastrointestinal leiomyosarcomas: CT features. Radiology 2003;227:385-390.

4. Tulasi NR, Kurian S, Mathew G, Viswanathan FR, Roul RK. Breast metastases from primary leiomyosarcoma. Aust NZ J Surg 1997;67:71-72

5. Lin CH, Yeh CN, Chen MF. Breast metastasis from uterine leiomyosarcoma: A case report. Arch Gynecol Obstet 2003;267:233-235.

6. Omeroglu G, Erssahin C, Potkul RK, Booth CN. FNA diagnosis of retroperitoneal leiomyosarcoma metastasizing to the breast. Diagn Cytopathol 2007;35(8):508-511.

7. Korman U, Ersavasti G, Kurugoglu S,Uygun NT, Akman C. Radiologic evaluation of gastrointestinal leiomyosarcomatosis. Eur Radiol 1997;7:1332-1334. 